\title{
Influence de la température sur le coefficient thermo-optique des fluorures alcalino-terreux
}

\author{
M. Lallemand et J. Martinet \\ Université de Poitiers, Ecole Nationale Supérieure de Mécanique et d'Aérotechnique, \\ rue Guillaume-VII, 86034 Poitiers Cedex, France
}

(Reçu le 10 avril 1981, révisé le 23 septembre 1981, accepté le 8 décembre 1981)

\begin{abstract}
Résumé. - On a mesuré par une méthode interférométrique les coefficients thermo-optiques $\mathrm{d} n / \mathrm{d} T$ de monocristaux de $\mathrm{CaF}_{2}, \mathrm{SrF}_{2}$, et $\mathrm{BaF}_{2}$ jusqu'à $500^{\circ} \mathrm{C}$ pour deux longueurs d'onde, 0,457 9/0,488 $0 \mu \mathrm{m}$ et 0,632 $8 \mu \mathrm{m}$, ainsi que leur coefficient de dilatation linéaire $\beta$ jusqu'à $350^{\circ} \mathrm{C}$.

Pour les trois solides on a observé une croissance linéaire de $\beta$ et une décroissance linéaire du coefficient (négatif) $\mathrm{d} n / \mathrm{d} T$ lorsque la température croît.

Une interprétation en terme de la variation de la polarisabilité électronique statique des ions avec la distance de séparation inter-ionique est proposée, qui rend compte avec succès de l'effet observé. On montre également à l'aide d'un modèle de polarisabilité dynamique classique à un seul oscillateur, que l'effet thermique sur $\mathrm{d} n / \mathrm{d} T$ peut être retrouvé dans le visible, en prenant un coefficient de largeur de bande interdite optique, dépendant linéairement de la température.
\end{abstract}

\begin{abstract}
By an interferometric method, the thermo-optic coefficients $\mathrm{d} n / \mathrm{d} T$ of single crystals of $\mathrm{CaF}_{2}, \mathrm{SrF}_{2}$ and $\mathrm{BaF}_{2}$ have been measured up to $500{ }^{\circ} \mathrm{C}$ for two wave lengths, $0.4579 / 0.4880 \mu \mathrm{m}$ and $0.6328 \mu \mathrm{m}$. The linear thermal expansion coefficient $\beta$ of these crystals have been also studied up to $350^{\circ} \mathrm{C}$.

The three solids show a linear increases of $\beta$ and a linear decrease of $\mathrm{d} n / \mathrm{d} T$, as the temperature raised.

An interpretation of the thermal effect on $\mathrm{d} n / \mathrm{d} T$ is proposed in terms of the static electronic polarizability variation of ions with the inter-ionic separation which agrees well with experimental data. With a dynamic polarizability model (single electronic oscillator), this effect is also reproduced, in the visible light, by taking a thermal gap coefficient with a linear temperature dependence.
\end{abstract}

L'indice de réfraction des cristaux ioniques et son coefficient de température ne sont connus à température élevée que pour un petit nombre de composés. Pour la structure cubique type $\mathrm{NaCl}$, Vishnievski et al. [1] ont effectué des mesures entre -150 et $+150^{\circ} \mathrm{C}$ dans le visible et le proche ultra-violet pour le fluorure de lithium, les chlorure et bromure de sodium et de potassium, ainsi que pour l'iodure de potassium. Feldman et al. [2] ont étudié l'évolution de $\mathrm{d} n / \mathrm{d} T$ du $\mathrm{LiF}, \mathrm{NaF}, \mathrm{KBr}, \mathrm{KCl}$ ainsi que les trois fluorures de métaux alcalino-terreux dans le rouge et l'infra-rouge entre -180 et $+180^{\circ} \mathrm{C}$. Les autres données existantes ont été en général obtenues au voişinage de la température ambiante [3].

La connaissance des valeurs des coefficients thermooptiques des cristaux semi-transparents à température élevée est cependant importante tant pour la recherche appliquée que fondamentale. C'est ainsi qu'elle permet de prévoir la distorsion des fronts d'onde des faisceaux laser se propageant dans un milieu présentant à la fois une absorption notable et une sensibilité de l'indice à la température (lentilles thermiques). Elle est nécessaire aussi dans la mise en ouvre de techniques expérimentales adaptées aux milieux semi-transparents, utilisées notamment en thermique (visualisation interférométrique, ombroscopie, strioscopie...).

$\mathrm{Du}$ point de vue fondamental ces informations expérimentales permettent, par exemple, de tester les théories concernant la variation de la polarisabilité électronique des ions avec la distance interionique dans un cristal.

L'influence de la température sur l'indice de réfraction d'un cristal ionique se manifeste de deux façons. Elle provoque des effets de densité directs, par l'intermédiaire de la dilatation du réseau cristallin et elle perturbe la structure de bandes électroniques (ou de la polarisabilité électronique des ions selon le schéma que l'on adopte); perturbations qui ont elles-mêmes 
deux composantes, l'une induite par les effets de densité, une autre à caractère intrinsèque se manifestant à densité constante.

Dans les cristaux appartenant à la structure fluorine, Schmidt et Vedam [4] en soumettant le cristal à l'influence d'une pression hydrostatique ont déterminé pour la raie $0,5896 \mu \mathrm{m}$ les coefficients de densité de la réfractivité de ces solides à la température ambiante; il est dès lors possible de séparer les effets liés à la densité des effets intrinsèques de température sur la structure électronique.

Nous présentons ici pour $\mathrm{CaF}_{2}, \mathrm{SrF}_{2}$ et $\mathrm{BaF}_{2}$ les résultats de mesure de leur coefficient thermo-optique $\mathrm{d} n / \mathrm{d} T$ dans un domaine de température s'étendant entre l'ambiante et $500^{\circ} \mathrm{C}$, pour les raies de longueurs d'onde 0,6328 et/ou 0,457 9-0,488 $0 \mu \mathrm{m}$.

Après avoir rappelé brièvement la technique expérimentale utilisée pour atteindre le coefficient de température de l'indice de réfraction ainsi que le coefficient de dilatation thermique du matériau, nous donnons au $\S 2$ les résultats de mesure de ces deux grandeurs. Par une première analyse, à caractère thermodynamique, de la fonction de Lorentz-Lorenz (L-L) nous déterminons au $\S 3$ le coefficient de température de la réfractivité à densité constante; dans une seconde analyse nous confrontons l'évolution thermique de la polarisabilité électronique moyenne des ions de ces composés avec les prévisions de la théorie des effets de proximité des ions dans le cristal, enfin nous effectuerons la comparaison avec les effets thermiques observés sur les spectres de réflexion dans l'ultra-violet.

1. Dispositif expérimental. - Dans notre étude sur le coefficient thermo-optique des verres de silicates sodo-calciques [5] nous avons décrit les méthodes interférométriques qui permettent d'atteindre d'une part le coefficient de température du chemin optique et d'autre part le coefficient de dilatation linéaire $\beta$ d'une lame épaisse à faces parallèles. Nous en rappellerons seulement ici les grandes lignes.

$\mathrm{Au}$ moyen d'un premier montage, nous avons étudié dans un four à effet Joule, l'évolution avec la température des franges de Fizeau apparaissant en lumière monochromatique dans une lame taillée en coin du matériau - cylindre de $20 \mathrm{~mm}$ de diamètre et $10 \mathrm{~mm}$ d'épaisseur. Ces premiers résultats fournissent, pour la longueur d'onde considérée, le coefficient de température du chemin optique du matériau $\mathrm{d} W / \mathrm{d} T=(\mathrm{d} n / \mathrm{d} T+n \beta)$.

Dans une seconde expérience on détermine le coefficient de dilatation thermique du solide à haute température. Dans ce but on place dans un four orientable l'éprouvette en position verticale que l'on dispose entre deux lames planes de silice fondue; dans l'espace annulaire entourant l'échantillon, entre les faces en regard, prend naissance un autre système de franges d'interférence, dont l'évolution avec la température permet d'accéder directement au coefficient de dilatation du matériau.
Dans ces conditions si d'une part $\Delta T$ et $\Delta T^{\prime}$ représentent respectivement les variations de température appliquées à l'échantillon dans le premier et le second essai (déterminés chacun par deux équilibres thermiques du four) et si d'autre par $\Delta S$ et $\Delta s$ sont les variations d'ordre d'interférence associées aux changements de chemin optique et à l'épaisseur du matériau, on a les relations

$$
\begin{aligned}
\frac{\Delta S}{\Delta T} & =\frac{2 L_{0}}{\lambda}\left(\frac{\mathrm{d} n}{\mathrm{~d} T}+n \beta\right) \\
\frac{\Delta s}{\Delta T^{\prime}} & =\frac{2 L_{0}}{\lambda} \beta
\end{aligned}
$$

où $L_{0}$ désigne l'épaisseur du cristal à la température ambiante, $\lambda$ la longueur d'onde de la radiation incidente, $n$ la valeur absolue de l'indice de réfraction du matériau à la température ordinaire.

Dans le premier montage l'échantillon est disposé au centre d'un épais cylindre de cuivre et un petit trou pratiqué dans l'éprouvette, permet de loger la tête d'un thermocouple dans le matériau. Dans le second dispositif, un thermocouple entoure seulement l'éprouvette. La précision sur la mesure de la température dans le premier cas est estimée à $\pm 0,2^{\circ} \mathrm{C}$ et dans le second cas à $\pm 0,3^{\circ} \mathrm{C}$. La température nominale de l'échantillon est prise comme la moyenne des températures entre les deux états d'équilibre thermique $\mathrm{du}$ four. Le comptage des franges est déterminé à $\pm 1 / 5$ de frange, de sorte que la précision sur $\beta$ est de $\pm 2 \%$ et celle sur $\mathrm{d} n / \mathrm{d} T$ de $\pm 4 \%$. En ce qui concerne le coefficient de température de la dispersion $\mathrm{d}^{2} n / \mathrm{d} T \mathrm{~d} \lambda$ la précision est de l'ordre de $4 \%$ également.

Les échantillons monocristallins provenaient de la société Sorem pour la fluorine (qualité u.v.), et de la Compagnie Harshaw pour $\mathrm{SrF}_{2}$ et $\mathrm{BaF}_{2}$.

2. Résultats. - L'évolution du coefficient de température du chemin optique $\mathrm{d} W / \mathrm{d} T$ en fonction de la température des trois composés ioniques a été représentée sur la figure 1 , pour les raies 0,4880 et $0,6328 \mu \mathrm{m}$ dans le cas de $\mathrm{CaF}_{2}$ et $\mathrm{SrF}_{2}$ et pour les raies 0,4579 et $0,6328 \mu \mathrm{m}$ pour $\mathrm{BaF}_{2}$. On peut observer que pour les trois matériaux la croissance de $\mathrm{d} W / \mathrm{d} T$ est linéaire dans tout le domaine de température exploré.

La variation du coefficient de dilatation linéaire des trois solides a été représentée sur la figure 2 entre l'ambiante et $350^{\circ} \mathrm{C}$. Pour $\mathrm{CaF}_{2}$ et $\mathrm{SrF}_{2}$ nous avons tenu compte pour le tracé de ces courbes de l'existence des données de Bailey et Hates [6] à $270 \mathrm{~K}$. En ce qui concerne $\mathrm{BaF}_{2}$ pour lequel il a été possible d'extrapoler nos valeurs jusqu'à cette température on constate un écart de $2 \%$ avec les données de ces auteurs. On a observé que la déviation des points de mesure par rapport à la courbe moyenne est au maximum de $\pm 2 \%$ sur tout le domaine de température. Dans le cas de $\mathrm{CaF}_{2}$ les valeurs de $\beta(T)$ obtenues peuvent être comparées à celles de Ballard et al. [7] sur de 


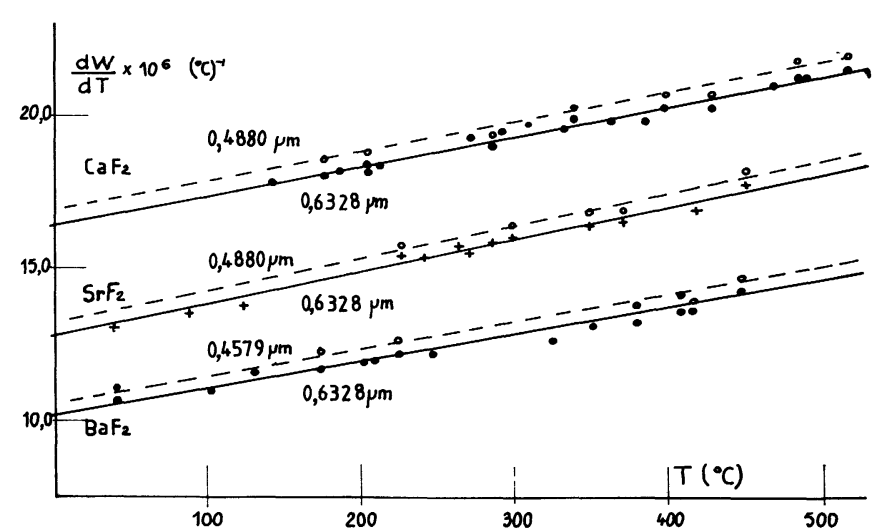

Fig. 1. - Evolution avec la température du trajet optique $\mathrm{d} W / \mathrm{d} T=(\mathrm{d} n / \mathrm{d} T+\beta n)$ des cristaux de $\mathrm{CaF}_{2}, \mathrm{SrF}_{2}$ et $\mathrm{BaF}_{2}$, pour deux longueurs d'ondes.

[Evolution with temperature of the optical length of single crystals of $\mathrm{CaF}_{2}, \mathrm{SrF}_{2}$ and $\mathrm{BaF}_{2}$ for two wave lengths.]

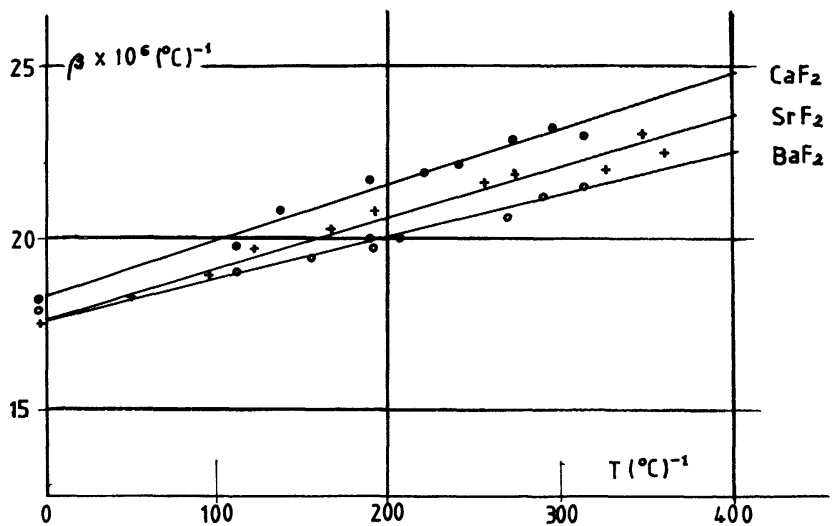

Fig. 2. - Variation du coefficient de dilatation linéaire de $\mathrm{CaF}_{2}, \mathrm{SrF}_{2}$ et $\mathrm{BaF}_{2}$ avec la température.

[Variation of the linear thermal expansion coefficient of $\mathrm{CaF}_{2}, \mathrm{SrF}_{2}$ and $\mathrm{BaF}_{2}$ versus temperature.]

Tableau I.

\begin{tabular}{|c|c|c|c|c|c|c|c|}
\hline Composés & $\lambda(\mu \mathrm{m})$ & $n\left(20^{\circ} \mathrm{C}\right)$ & $\begin{array}{l}\beta\left(20^{\circ} \mathrm{C}\right) \\
\times 10^{6}{ }^{\circ} \mathrm{C}^{-1}\end{array}$ & $\begin{array}{c}\mathrm{d} \beta / \mathrm{d} T \\
\times 10^{6}{ }^{\circ} \mathrm{C}^{-1}\end{array}$ & $\begin{array}{r}\mathrm{d} n / \mathrm{d} T\left(20^{\circ} \mathrm{C}\right) \\
\times 10^{6}{ }^{\circ} \mathrm{C}^{-1}\end{array}$ & $\begin{array}{l}\mathrm{d}^{2} n / \mathrm{d} T^{2} \\
\times 10^{8}{ }^{\circ} \mathrm{C}^{-2}\end{array}$ & $\begin{array}{c}\mathrm{d}^{2} n / \mathrm{d} T \mathrm{~d} \lambda \\
\times 10^{6}{ }^{\circ} \mathrm{C}^{-1} \mu \mathrm{m}^{-1}\end{array}$ \\
\hline$\overline{\mathrm{CaF}_{2}}$ & $\begin{array}{r}\overline{4} \\
0,4880 \\
0,6328\end{array}$ & $\begin{array}{r}-\overline{7} \\
1,43720 \\
1,43329\end{array}$ & $18,7 \pm 0,3$ & $\overline{1,60}$ & $\begin{array}{r}-\overline{-} \pm 0,5 \\
-\quad 9,9 \pm 0,5 \\
-10,2 \pm 0,5\end{array}$ & $-\overline{1,31}$ & $-2, \overline{1} \pm 0,1$ \\
\hline $\mathrm{SrF}_{2}$ & $\begin{array}{l}0,4880 \\
0,6328\end{array}$ & $\begin{array}{l}1,44126 \\
1,43707\end{array}$ & $17,9 \pm 0,3$ & 1,48 & $\begin{array}{l}-12,4 \pm 0,5 \\
-12,7 \pm 0,5\end{array}$ & $-1,08$ & $-2,1 \pm 0,1$ \\
\hline $\mathrm{BaF}_{2}$ & $\begin{array}{l}0,4579 \\
0,6328\end{array}$ & $\begin{array}{l}1,48020 \\
1,47327\end{array}$ & $17,9 \pm 0,3$ & 1,21 & $\begin{array}{l}-15,7 \pm 0,5 \\
-16,0 \pm 0,5\end{array}$ & $-0,87$ & $-1,7 \pm 0,1$ \\
\hline
\end{tabular}

l'Irtran entre 40 et $240{ }^{\circ} \mathrm{C}$, l'écart de $2 \%$ observé à $20^{\circ} \mathrm{C}$ se réduit à $0,5 \%$ à $240^{\circ} \mathrm{C}$. En revanche les pentes des courbes $\beta(T)$ à basses températures de Bayley et Yates, extrapolée au-delà de la température ambiante, diffèrent fortement des nôtres.

La connaissance des courbes de variation en fonction de la température de $\mathrm{d} W / \mathrm{d} T$ et de $\beta(T)$ (celles-ci extrapolées au-delà de $350^{\circ} \mathrm{C}$ ) nous a permis d'accéder à l'évolution du coefficient $\mathrm{d} n / \mathrm{d} T$ de $\mathrm{CaF}_{2}, \mathrm{SrF}_{2}$ et $\mathrm{BaF}_{2}$ jusqu'à $500^{\circ} \mathrm{C}$ pour les différentes longueurs d'onde du visible mentionnées, ainsi que de déterminer le coefficient de température de la dispersion dans ce domaine. Ces résultats sont contenus dans les tableaux I et II.

La courbe de variation du coefficient $\mathrm{d} n / \mathrm{d} T$ en fonction de la température des trois fluorures alcalinoterreux a été représentée sur la figure 3. On note pour chaque longueur d'onde la décroissance linéaire de ce coefficient (négatif dans le visible) lorsque $T$ augmente et le faible effet de la dispersion dans cette région du spectre. Pour $\mathrm{CaF}_{2}$ les résultats à la température de $40^{\circ} \mathrm{C}$ de Lipson et al. [8], de Harris et al. [9] ainsi que

Tableau II.

$$
\lambda=0,6328(\mu \mathrm{m})
$$

\begin{tabular}{|c|c|c|c|c|c|c|c|}
\hline Cristaux & $T\left({ }^{\circ} \mathrm{C}\right)$ & $\begin{array}{c}\mathrm{d} n / \mathrm{d} T \\
\times 10^{6}{ }^{\circ} \mathrm{C}^{-1}\end{array}$ & $\begin{array}{c}(\rho \partial n / \partial \rho) \\
{[4]}\end{array}$ & $\begin{array}{c}3 \beta \\
\times 10^{6}{ }^{\circ} \mathrm{C}^{-1}\end{array}$ & $\left(\frac{\rho}{R} \frac{\partial R}{\partial \rho}\right)_{T}^{\mathrm{LL}}$ & $\begin{array}{c}\frac{1}{R} \frac{\mathrm{d} R}{\mathrm{~d} T} \\
\times 10^{6}{ }^{\circ} \mathrm{C}^{-1}\end{array}$ & $\begin{array}{l}\left(\frac{1}{R} \frac{\partial R}{\partial T}\right)_{\rho} \\
\times 10^{6}{ }^{\circ} C^{-1}\end{array}$ \\
\hline - & - & - & - & - & - & - & - \\
\hline $\mathrm{CaF}_{2}$ & $\begin{array}{r}20 \\
500\end{array}$ & $\begin{array}{l}-10,2 \\
-16,5\end{array}$ & 0,208 & $\begin{array}{l}56,1 \\
79,2\end{array}$ & $-0,581$ & $\begin{array}{l}35,6 \\
46,0\end{array}$ & $\begin{array}{l}3,0 \\
0,0\end{array}$ \\
\hline $\mathrm{SrF}_{2}$ & $\begin{array}{r}20 \\
500\end{array}$ & $\begin{array}{l}-12,7 \\
-17,9\end{array}$ & 0,244 & $\begin{array}{l}53,7 \\
75,0\end{array}$ & $-0,511$ & $\begin{array}{l}28,2 \\
39,1\end{array}$ & $\begin{array}{l}0,8 \\
0,8\end{array}$ \\
\hline $\mathrm{BaF}_{2}$ & $\begin{array}{r}20 \\
500\end{array}$ & $\begin{array}{l}-16,0 \\
-20,2\end{array}$ & 0,322 & $\begin{array}{l}53,7 \\
71,1\end{array}$ & $-0,417$ & $\begin{array}{l}24,7 \\
34,5\end{array}$ & $\begin{array}{l}2,3 \\
4,9\end{array}$ \\
\hline
\end{tabular}




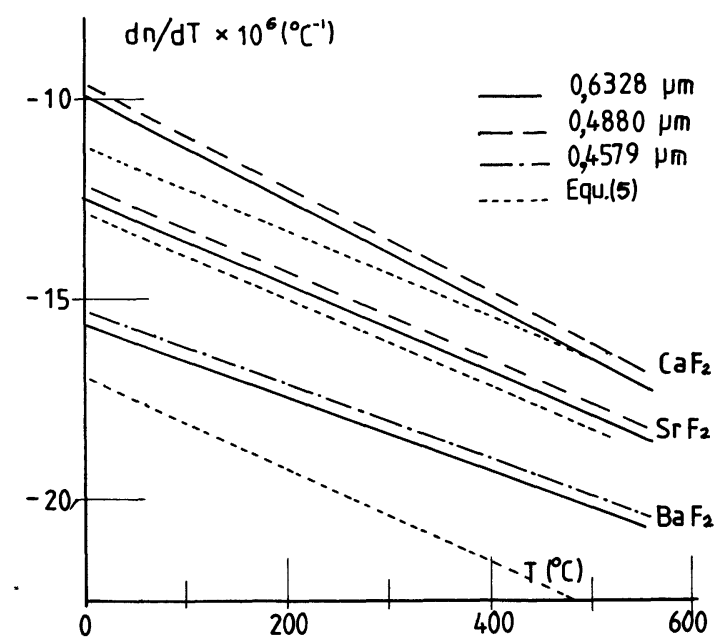

Fig. 3. - Variation de $\mathrm{d} n / \mathrm{d} T$ avec la température de $\mathrm{CaF}_{2}, \mathrm{SrF}_{2}$ et $\mathrm{BaF}_{2}$ pour deux longueurs d'onde du visible. Trait (----), équation (5).

[Variation of $\mathrm{d} n / \mathrm{d} T$ of $\mathrm{CaF}_{2}, \mathrm{SrF}_{2}$ and $\mathrm{BaF}_{2}$ single crystals in function of temperature, for two wave lengths of the visible. Broken line (-----), equation (5).]

de Feldman et al. [2] s'écartent des nôtres d'environ $-9 \%$; cette différence étant attribuable selon nous aux valeurs du coefficient de dilatation linéaire distinctes des nôtres prises par ces auteurs qui tous mettent en œuvre la méthode interférométrique. En revanche, un bon accord est enregistré à la température ambiante avec Malitson [10] dont les mesures ont été effectuées au moyen de la méthode de déviation du prisme laquelle ne nécessite aucune mesure complémentaire de la dilatation. Pour $\mathrm{SrF}_{2}$ et $\mathrm{BaF}_{2}$ à $40^{\circ} \mathrm{C}$, les valeurs $\mathrm{de} \mathrm{d} n / \mathrm{d} T$ obtenues ici sont en accord satisfaisant avec celles de la littérature [2]; en ce qui concerne $\mathrm{BaF}_{2}$, les résultats à $25^{\circ} \mathrm{C}$ de Malitson [11] ne présentent une différence que de $-5 \%$ avec les nôtres.

La comparaison des résultats obtenus pour $\mathrm{d} n / \mathrm{d} T$ à température élevée avec les données déjà publiées est limitée à $180^{\circ} \mathrm{C}$ avec les mesures de Feldman et al. [2]; pour la raie $0,6328 \mu \mathrm{m}$ les différences enregistrées sont de $-11 \%$ pour $\mathrm{CaF}_{2},+6 \%$ pour $\mathrm{SrF}_{2}$ et de $-6 \%$ pour $\mathrm{BaF}_{2}$.

3. Discussion. - L'analyse de l'effet de température sur le coefficient thermo-optique des cristaux ioniques peut s'effectuer selon plusieurs points de vue. Dans une première approche, à caractère thermodynamique, il est possible de déduire par exemple des quantités comme le coefficient de température de la réfractivité du cristal à densité constante et de montrer l'existence de corrélations entre les grandeurs mesurées, coefficient de température et coefficient de densité de l'indice. Une seconde approche en terme de polarisabilité électronique des ions offre la possibilité de tester la théorie semi-empirique de Jai Shankar et al. [12] de l'influence des interactions ioniques sur ce paramètre. Enfin l'analyse peut être menée en terme d'évolution avec la température de la largeur de bande de transitions électroniques interdite à l'aide du modèle TBM, proposé par Tsay, Bendow et Mitra [13]. Ces deux dernières représentations qui mettent en jeu, l'une, des objets en interaction, l'autre, des effets collectifs, peuvent d'ailleurs être considérées comme équivalentes lorsqu'on introduit le modèle de Drude pour la polarisabilité dynamique des ions.

On sait que le modèle de Lorentz-Lorenz est plus apte à rendre compte des propriétés diélectriques des cristaux ioniques cubiques que celui de Drude; il dérive, en effet, d'une expression du champ électrique effectif de Lorentz qui est rigoureuse pour une distribution de la densité électronique fortement localisée alors que le modèle de Drude n'est valable que pour une distribution étendue de charge [14]. Nous utiliserons donc ici la formule de Lorentz-Lorenz pour représenter l'équation d'état diélectrique de nos solides. Nous supposerons de plus que pour la structure fluorine chaque molécule du cristal $\left(\mathrm{Ca}^{+2}, 2 \mathrm{~F}^{-}\right)$possède une polarisabilité dynamique $\alpha_{m}$ donnée par la règle d'additivité des polarisabilités individuelles des ions.

Dans ces conditions, pour une température et une pression fixées, la variation du carré de l'indice de réfraction avec la densité peut s'écrire

$$
\mathrm{L}-\mathrm{L}=\frac{n^{2}-1}{n^{2}+2} \frac{1}{\rho}=R(\omega)
$$

où $\rho$ est le nombre de molécules par unité de volume et $R(\omega)$ la réfractivité moléculaire du solide à la fréquence $\omega$.

3.1 INTERPRÉTATION THERMODYNAMIQUE. - En prenant la dérivée par rapport à la température de l'expression (1), le coefficient de température de l'indice s'écrit

$$
\frac{\mathrm{d} n}{\mathrm{~d} T}=\frac{\left(n^{2}-1\right)\left(n^{2}+2\right)}{6 n}\left(-3 \beta+\frac{1}{R} \frac{\mathrm{d} R}{\mathrm{dT}}\right) .
$$

On observe que l'évolution de $\mathrm{d} n / \mathrm{d} T$ avec la température résulte de la compétition entre deux effets, l'un purement thermique lié à la variation de densité du cristal, un autre dû à l'influence de la température sur la réfractivité. Le coefficient de température de la réfractivité se compose lui-même de deux contributions, en effet on peut écrire

$$
\frac{1}{R} \frac{\mathrm{d} R}{\mathrm{~d} T}=\left(\frac{1}{R} \frac{\partial R}{\partial T}\right)_{\rho}-3 \beta\left(\frac{\rho}{R} \frac{\partial R}{\partial \rho}\right)_{T}
$$

le premier terme exprimant la dépendance intrinsèque en la température de la réfractivité à volume constant, le second, les effets induits par la dilatation thermique - déplacement mutuel des ions. De sorte que

$$
\begin{aligned}
\frac{\mathrm{d} n}{\mathrm{~d} T}= & \frac{\left(n^{2}-1\right)\left(n^{2}+2\right)}{6 n} \times \\
& \times\left[-3 \beta+\left(\frac{1}{R} \frac{\partial R}{\partial T}\right)_{\rho}-3 \beta\left(\frac{\rho}{R} \frac{\partial R}{\partial \rho}\right)_{T}\right]
\end{aligned}
$$


Mais la dérivation par rapport à la densité de la fonction L-L nous donne également

$$
\left(\rho \frac{\partial n}{\partial \rho}\right)_{T}=\frac{\left(n^{2}-1\right)\left(n^{2}+2\right)}{6 n}\left[1+\left(\frac{\rho}{R} \frac{\partial R}{\partial \rho}\right)_{T}\right] .
$$

Le coefficient $\left(\frac{\rho}{R} \frac{\partial R}{\partial \rho}\right)_{T}$ de (4) peut donc se déduire directement du coefficient de densité de l'indice. Comme nous l'avons signalé cette quantité est connue expérimentalement pour les fluorures alcalino-terreux [4]. Dans le tableau II nous avons reporté les valeurs de ce coefficient ainsi que celles déduites de $\left(\frac{\rho}{R} \frac{\partial R}{\partial \rho}\right)_{T}$; on notera le signe toujours négatif de ce dernier, correspondant donc à la décroissance de la réfractivité lorsque la densité augmente, phénomène que l'on observe d'une façon générale dans les milieux condensés [15].

Dans ce qui suit nous avons admis que pour les trois cristaux, le coefficient $\left(\frac{\rho}{R} \frac{\partial R}{\partial \rho}\right)_{T}$ restait invariant avec la température. Dans ces conditions, compte tenu de nos données expérimentales sur $\mathrm{dn} / \mathrm{d} T$ et $\beta(T)$, l'expression (3) permet d'accéder aux deux coefficients $\frac{1}{R} \frac{\mathrm{d} R}{\mathrm{~d} T}$ et $\left(\frac{1}{R} \frac{\partial R}{\partial T}\right)_{\rho}$ (voir tableau II). On constate que pour chaque composé le coefficient de température total $\frac{1}{R} \frac{\mathrm{d} R}{\mathrm{~d} T}$ augmente avec la température et est plus faible pour les cristaux formés de cations lourds.

A partir de nos résultats, nous pouvons aussi calculer l'évolution thermique de la polarisabilité électronique, en valeur absolue, pour les trois solides, en nous référant aux valeurs fiduciaires de l'indice et de la densité à température ambiante (voir tableau I). Pour cela nous avons évalué entre l'ambiante et $500^{\circ} \mathrm{C}$ la fonction L-L qui présente pour les trois fluorures alcalino-terreux une croissance linéaire avec la densité du solide. Les valeurs de $\alpha_{m}$ déterminées pour la raie $0,6328 \mu \mathrm{m}$ sont les suivantes :

\begin{tabular}{ccc} 
Composés & \multicolumn{2}{c}{$\alpha_{m}\left(\AA^{3}\right)$} \\
- & $20{ }^{\circ} \mathrm{C}$ & $500^{\circ} \mathrm{C}$ \\
$\mathrm{CaF}_{2}$ & 2,53 & 2,58 \\
$\mathrm{SrF}_{2}$ & 3,05 & 3,09 \\
$\mathrm{BaF}_{2}$ & 4,00 & 4,05
\end{tabular}

Les valeurs à $20^{\circ} \mathrm{C}$ sont en accord avec celles de Tesman et al. [16].

Retournant au tableau II, nous voyons que les valeurs du coefficient de température de la réfractivité à densité constante sont faibles pour les trois solides, s'annulant même dans le cas de $\mathrm{CaF}_{2}$ à haute température. Nous retrouvons la remarque énoncée il y a plus de trente ans par Ramachandra [17] d'après l'analyse des coefficients de température $\mathrm{d} n / \mathrm{d} T$ connus à cette époque : l'effet purement thermique (à densité constante) sur la polarisabilité moyenne des ions des cristaux ioniques est bien plus faible que celui provoqué par la variation du réseau.

Ce résultat a une conséquence importante, en effet il entraîne la relation approximative

$$
\frac{\mathrm{d} n}{\mathrm{~d} T}=-3 \beta\left(\rho \frac{\partial n}{\partial \rho}\right)_{T}
$$

qui corrèle directement le coefficient de température de l'indice d'un matériau à son coefficient de densité et à celui de la dilatation thermique, relation fréquemment utilisée pour sa simplicité. Dans le cas de $\mathrm{CaF}_{2}$ et $\mathrm{SrF}_{2}$ elle conduit à des résultats très bien vérifiés par l'expérience dans tout le domaine de température exploré; elle s'applique moins bien au cas de $\mathrm{BaF}_{2}$ pour lequel l'approximation faite est trop forte (voir Fig. 3).

3.2 INFLUENCE DES INTERACTIONS IONIQUES SUR LA POLARISABILITÉ ÉLECTRONIQUE DES IONS. - La formulation du coefficient $d n / d T$ en fonction du coefficient de température de la réfractivité du cristal se prête bien à une approche théorique de l'évolution thermique de $\mathrm{d} n / \mathrm{d} T$ des cristaux ioniques en terme de la variation de la polarisabilité électronique des ions avec la distance de séparation inter-ionique.

En désignant par $a$ la distance inter-ionique on peut réécrire (2) sous la forme

$$
\frac{\mathrm{d} n}{\mathrm{~d} T}=\frac{\left(n^{2}-1\right)\left(n^{2}+2\right)}{6 n} 3 \beta\left[-1+\frac{1}{3} \frac{a}{\alpha_{m}} \frac{\mathrm{d} \alpha_{m}}{\mathrm{~d} a}\right]
$$

où apparaissent factorisés les effets dus à la dilatation thermique et ceux de réseau sur la polarisabilité du cristal. Le coefficient $\frac{a}{\alpha_{m}} \frac{\mathrm{d} \alpha_{m}}{\mathrm{~d} a}$ a été récemment calculé par Jai Shankar et al. [12] à partir de considérations élémentaires basées sur la corrélation existante entre la polarisabilité électronique des ions et leur rayon ionique.

Vedam et al. [4] avaient déjà fait remarquer empiriquement que les valeurs des polarisabilités électroniques des cations et des anions d'un cristal ionique, respectivement croissaient et décroissaient, lorsque ceux-ci passent de l'état libre à l'état condensé dans le cristal; un comportement analogue étant observé pour les rayons ioniques. Postulant une relation fonctionnelle entre rayon et polarisabilité des espèces ioniques du type $\alpha_{i}=k r_{i}^{p}$, Jai Shankar et al. ont déterminé à partir des valeurs empiriques tabulées de $\alpha_{\mathrm{i}}$ et de $r_{\mathrm{i}}$ les coefficients inconnus. Dans ces conditions, les variations de $\alpha_{i}$ avec la distance interionique $a$ peuvent être calculées si l'on possède la loi de variation de $r_{\mathrm{i}}$ avec $a$. Or on connaît cette loi, c'est une fonction de l'énergie moyenne d'excitation des ions à l'état libre modulée par l'énergie de réseau de Madelung, qui dépend explicitement de $a$. Les valeurs du coefficient $\frac{1}{3} \frac{a}{\alpha_{m}} \frac{\mathrm{d} \alpha_{m}}{\mathrm{~d} T}$ obtenu par ces auteurs pour la struc- 
Tableau III.

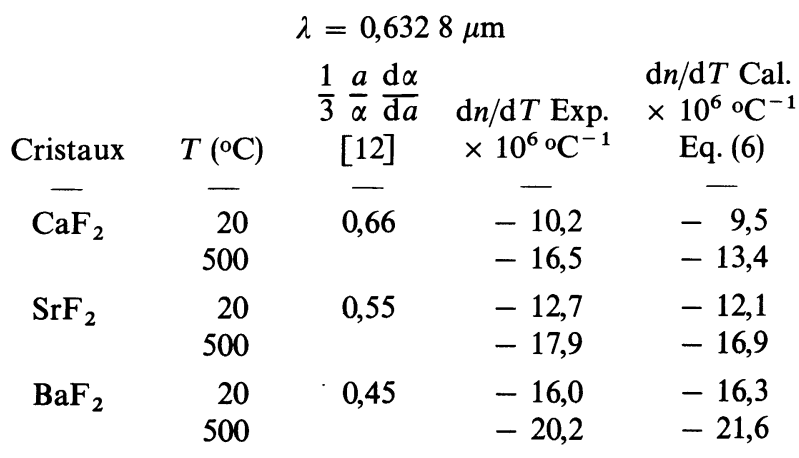

ture fluorine ont été reportées dans le tableau III. Nous $\mathrm{y}$ avons inclus les valeurs de $\mathrm{d} n / \mathrm{d} T$ calculées à partir de l'expression (6) pour les deux températures 20 et $500^{\circ} \mathrm{C}$.

On constate que pour les trois composés ioniques d'une part le signe de l'effet de température prévu par cette théorie pour $\mathrm{d} n / \mathrm{d} T$ est correct, mais que d'autre part pour la raie $0,6328 \mu \mathrm{m}$ les valeurs absolues sont en bon accord avec l'expérience à $20^{\circ} \mathrm{C}$, elles s'en écartent légèrement à $500^{\circ} \mathrm{C}$, excepté pour $\mathrm{CaF}_{2}$ pour lequel une différence plus forte est constatée.

3.3 Comparaison AVEC LE MODÈle DE BANDE. Le modèle de polarisabilité dynamique de Drude à un seul oscillateur permet d'écrire

$$
\alpha_{m}(\omega)=\frac{e^{2}}{m} \frac{1}{\omega_{\mathbf{g}}^{2}-\omega^{2}}
$$

où $\omega_{\mathrm{g}}$ désigne la fréquence de transition fondamentale du modèle sans amortissement.

Dans ces conditions, en introduisant le coefficient de température de la polarisabilité moléculaire dans l'expression (2) donnant le coefficient thermo-optique on obtient :

$\frac{\mathrm{d} n}{\mathrm{~d} T}=\frac{\left(n^{2}-1\right)\left(n^{2}+2\right)}{6 n} \times$

$$
\times\left(-3 \beta-2 \frac{\mathrm{d} \log \omega_{\mathrm{g}}}{\mathrm{d} T} \frac{1}{1-\frac{\omega^{2}}{\omega_{\mathrm{g}}^{2}}}\right)
$$

Le modèle TBM de Tsay et al. [13] à un oscillateur électronique contient les mêmes éléments, il diffère de l'expression (8) par un terme $\left(n^{2}-1\right) / 2 n$ que remplace $\operatorname{ici}\left(n^{2}-1\right)\left(n^{2}+2\right) / 6 n$. Pour une fréquence fixée l'évolution de $\mathrm{d} n / \mathrm{d} T$ avec la température résulte comme précédemment de la variation de deux termes l'un de nature purement thermique, l'autre associé aux modifications de la largeur de bande interdite des transitions électroniques.

La considération de la formule de dispersion (8) et de nos résultats expérimentaux pour les longueurs d'onde étudiées, nous ont permis de déduire, par une analyse graphique, les deux éléments intéressants du modèle, l'évolution du coefficient de température de la largeur de bande interdite et les fréquences de transitions fondamentales $\omega_{\mathrm{g}}$ des cristaux. Nous avons consigné ces résultats dans le tableau IV.

On remarque que les valeurs de $\omega_{\mathrm{g}}$ inférées sont toutes très voisines des premiers pics intenses de réflexion observés par Rubloff [18] dans les spectres en ultra-violet lointain des trois composés et identifiés comme transitions excitonique et interbande $\left(\Gamma_{15}-\Gamma_{1}\right)$. Si l'on applique à cette analyse le modèle TBM original, des valeurs nettement plus faibles de $\omega_{\mathrm{g}}$ sont obtenues; ceci justifie l'utilisation de la formule de LorentzLorenz.

En ce qui concerne le coefficient $\frac{\mathrm{d} \log \omega_{\mathrm{g}}}{\mathrm{d} T}$ les résultats de l'analyse par le modèle de bande sont en accord en sens et en amplitude avec le déplacement vers le rouge des pics du spectre électronique que les expériences de Rubloff ont mis en évidence, sous l'influence

\begin{tabular}{|c|c|c|c|c|}
\hline \multirow[t]{2}{*}{ Cristaux } & \multicolumn{2}{|r|}{$\omega_{\mathrm{g}}\left(\mathrm{cm}^{-1}\right)$} & \multicolumn{2}{|c|}{$-\mathrm{d} \log \omega_{\mathrm{g}} / \mathrm{d} T\left({ }^{\circ} \mathrm{C}^{-1}\right)$} \\
\hline & Eq. (8) & Pics de réflexion [18] & Eq. (8) & $\begin{array}{c}\text { Déplacement pics réflexion } \\
{[18]}\end{array}$ \\
\hline $\mathrm{CaF}_{2}$ & 89000 & 90200,97600 & $1,7 \times 10^{-5}+1,0 \times 10^{-8} T$ & $1,5 \times 10^{-4}, 3 \times 10^{-5}$ \\
\hline $\mathrm{SrF}_{2}$ & 83500 & 85500,90700 & $1,4 \times 10^{-5}+1,0 \times 10^{-8} T$ & $1,3 \times 10^{-4}, 6 \times 10^{-5}$ \\
\hline $\mathrm{BaF}_{2}$ & 78000 & 80700,85300 & $1,2 \times 10^{-5}+1,0 \times 10^{-8} T$ & $4,0 \times 10^{-5}$ \\
\hline
\end{tabular}
d'une élévation de la température.

Tableau IV. 


\section{Bibliographie}

[1] VishnevskiI, V. N., KuZyK, M. P. and StefanskiI, I. V., Sov. Phys. Solid State 17 (1976) 2110-2112.

[2] Feldman, A., Horowitz, D. and Waxler, R. M., NBS Special Publication 1977, no 509, STP 655 ASTM, 74-81.

[3] LI, H. H., J. Phys. Chem. Ref. Data 5 (1976) 528.

[4] Schmidt, E. D. D. and Vedam, K., J. Phys. Chem. Solids 27 (1966) 1563-1566.

Schmidt, E. D. D., Thesis, Pennsylvania Univ. (1972).

[5] Lallemand, M. et Martinet, J., Verres Réfract. 35 (1981) 675-680.

[6] Bailey, A. C. and Yates, B., Proc. Phys. Soc. 91 (1967) 390-395.

[7] Ballard, S. S., Brown, S. E. and Browder, T. S., Appl. Opt. 17 (1978) 1152-1154.

[8] Lipson, H. G., TsAY, Y. F., Bendow, B. and Logor, P. A., Appl. Opt. 15 (1976) 2352-2354.
[9] Harris, R. J., Johnston, G. T., KrohQ, G. A. and Mukai, H., Appl. Opt. 16 (1977) 436-438.

[10] Malitson, I. H., Appl. Opt. 2 (1963) 1103-1107.

[11] Malitson, I. H., J. Opt. Soc. Am. 54 (1964) 628-632.

[12] JaI Shanker, LashkaU, A. K. G. and Sharma, O. P., Indian J. Pure Appl. Phys. 16 (1978) 578-581.

[13] Tsay, Y. F., Bendow, B. and Mitra, S. S., Phys. Rev. B 8 (1973) 2688-2696.

[14] Guertin, R. F. and Stern, F., Phys. Rev. 134A (1964) 427-433.

[15] Lallemand, M. et Vidal, D., J. Chem. Phys. 66 (1977) 4776-4780.

[16] Tesman, J. R., Kahn, A. H. and Shockley, W., Phys. Rev. 92 (1953) 890-895.

[17] Ramachandra, G. N., Proc. Indian Acad. Sci. 25A (1947) 208.

[18] Rubloff, G. W., Phys. Rev. B 5 (1972) 662-679. 